\title{
Intuición y concepto. Ampliación simmeliana de la epistemología de Nietzsche
}

\author{
Reynner Franco \\ Departamento de Filosofía y Lógica y Filosofía de la Ciencia \\ Universidad de Salamanca \\ rfranco@usal.es
}

La actividad del entendimiento es vida.

Aristóteles Metafísica XII, 7, $1072 b 26$

El espíritu humano por naturaleza, es inclinado a las abstracciones y considera como estable lo que está en continuo cambio. F. Bacon, Novum organum, libro I, § 45

'Toda conceptualización es abstracción' - en su sentido más estéril. Así podríamos resumir uno de los principales resultados epistemológicos de Sobre verdad y mentira, personificada en el «hombre racional», quien posee la «capacidad de volatilizar las metáforas intuitivas en un esquema; en suma, [...] de disolver una figura en un concepto» ${ }^{1}$. Sin duda Nietzsche es uno de los pensadores más incontestables al respecto, lo cual se refleja, entre otras cosas, en el hecho de que el pensamiento actual deba gran parte de sus tesis ontológicas, hermenéuticas, analíticas, pragmáticas y morales a planteamientos y críticas centrales de su filosofía ${ }^{2}$. Hay suficiente

${ }^{1}$ Friedrich Nietzsche, Sobre verdad y mentira en sentido extramoral (=VM), trads. L. M. Valdés y T. Orduña, Madrid: Tecnos 2007, p. 26.

2 Un análisis sucinto y actual de la influencia y crítica de los conceptos de voluntad de poder, relativismo y totalitarismo en el postmodernismo y la Escuela de Frankfurt se encuentra en H. C. F. Mansilla, «Friedrich Nietzsche, la Escuela de Frankfurt y el postmodernismo», Revista de Filosofía 51 (2005), 73-97 pp. 
literatura al respecto, aunque se echan en falta referencias más detenidas a una recepción que en su día, reconociendo la importancia y atingencia de los planteamientos nietzscheanos, ensayara ya una manera de incorporar las exigencias de Nietzsche a los aspectos que quedaban fuera - en tanto criticados o reducidos - de los pilares de su filosofía. Me refiero a la recepción de G. Simmel ${ }^{3}$.

En el campo epistemológico, el 'alambique' que elaboró Nietzsche para destilar lo racional de lo intuitivo puede producir no sólo un licor embriagador y delirante, sino también un buen digestivo. Simmel incorpora ramificaciones que abren la posibilidad de argumentar, sin dejar de ser nietzscheano, que las metáforas, metonimias o abstracciones de lo conceptual son modos de desarrollar intuiciones no menos inmediatas respecto a su origen vital, o mejor, que ejecutan sus operaciones con las mismas 'constantes vitales' de un 'organismo' que se mantiene y supera a sí mismo. Hablo de 'origen vital' de estos 'modos de vida' - según nuestra lectura de Simmel-, porque, a juzgar por su exposición en Lebensanschauung, el concepto orgánico de vida ocupa un lugar central - lo que forma parte del espíritu del momento ${ }^{4}-$, sin embargo no parece ser suficiente para concebir desde sí su propia esencia y autoorganización.

Simmel acude por tanto a registros vitalistas, perspectivistas y dialécticos (límite, voluntad, tiempo y autoconciencia) como operadores de una intuición más amplia de la vida que sitúa a lo conceptual en el mismo plano necesario de lo intuitivo, en tanto su continuidad y realización de sus impulsos. Una epistemología intuicionista que no se limita a la orientación instintiva básica, pues

3 Sobre la recepción simmeliana del concepto de vida de Nietzsche en relación con la filosofía moral y estética, cf. D. Solies, «"Mit den besseren Mitteln die schlechtere Sache verteidigen". Georg Simmels Rezeption von Nietzsches Lebensbegriff», en: R. ReschKe (ed.), Zeitenwende - Wertewende. Internationaler Kongress der Nietzsche-Gesellschaft zum 100. Todestag Friedrich Nietzsches, Berlin: Akademie Verlag 2001, 177-180 pp.

4 G. Simmel, Der Konflikt der modernen Kultur:(KmK), en: GSG 16, 1999, 188. O también Simmel, SchNiet (GSG 10), 187-189. Simmel apunta que en la Europa intelectual de comienzos del s. XX «el concepto de vida aspira al lugar central en el que la realidad de los valores - metafísicos y psicológicos, éticos y artísticos - tienen su punto de partida y encuentro» (Simmel, KmK [GSG 16], 188). 
el acto de intuir y su contenido parecen disfrutar ambos de cierta autonomía, aunque su resultado sea tenido como ilusorio. Los contenidos de las representaciones poseen por tanto estructuras y funcionalidades correspondientes al fenómeno vital, en tanto su utilidad - secundando a Nietzsche, y en contra de la «posibilidad» kantiana - engendra los objetos del conocimiento, tesis desde la que Simmel ensaya «una relación entre selección natural y teoría del conocimiento» ${ }^{5}$ y que se mantiene en su programa sistemático más completo de Intuición de la vida .

Gran parte del programa de Simmel se encuentra presente ya en Nietzsche, aunque con algunas diferencias. Que «la fuerza de los conocimientos» resida «en su antigüedad, en su incorporación, en su carácter de condición para la vida ${ }^{7}$ y que su origen y desarrollo sólo sean resultado de un «juego instintivo intelectual, inocente y feliz», cuyas referencias iniciales, como casos excepcionales -así Nietzsche-, se encuentran en los planteamientos eléatas y escépti-

${ }^{5}$ Cf. G. Simmel, Über eine Beziehung der Selektionslehre zur Erkenntnistheorie:(=Übse) en: Archiv für systematische Philosophie 1 (1895), 34-45 pp. (repr. en: Simmel, Zur Philosophie der Kunst - Philosophische und kunstphilosophische Aufsätze, Potsdam: Kiepenheuer 1992, 111-125 pp.).

${ }^{6}$ El viejo artículo de Jankélévitch, recientemente traducido, recuerda el carácter contradictorio necesario que Simmel observó al respecto - anotado por Rickert para refutar la posibilidad de sintetizar las dos exigencias contradictorias de la forma y la vida, la estructura de la autotrascendencia-: «primero, que la forma, en su calidad de límite, de principio de individualización, es necesaria al pensamiento conceptual y discursivo; la ciencia, por sus razonamientos, por sus definiciones, postula impresionantemente ese elemento estable e intemporal. Y, por consiguiente, el hecho de postular una forma viviente y variable, un límite dilatable, equivale, como Simmel reconoce a menudo, a salir de nuestra lógica común fundada en el principio de identidad», en: V. JANKÉLÉvitch, Georg Simmel, filósofo de la vida, Barcelona: Gedisa 2007, p. 87. [«Georg Simmel, philosophe de la vie», Revue de Métaphysique et de Morale 32 (1925), 213-257 pp., 303-386 pp.]. Problema que, desde luego, está presente en Simmel, aunque sin la perplejidad ante la contradicción, pues justamente el concepto de forma no puede mantener su estabilidad si se considera la motilidad de la vida como «raíz de la lógica», cuya forma es dinamismo - subyacente al reposo (cf., p. 89) - . Para una aproximación al carácter dialéctico de la forma autotrascendente de la vida en Simmel bajo el modelo del movimiento de la autoconciencia, cf. R. Franco, «Ontologie des Lebens als unmittelbare Wahrheit des Denkens. Anmerkungen zu Hegels Leben-Erkennen-Dialektik und Simmels Selbsttranszendenz des Lebens», Hegel-Jahrbuch 2007 (Das Leben denken II), 206-212 pp.

7 Friedrich Nietzsche, Die fröhliche Wissenschaft (=FW), en KSA 3, 1980 §110, p. 469. 
$\cos ^{8}$, todo ello, y más, muestra que el único criterio válido para hablar de conocimiento en general $-\mathrm{y}$ de concepto en particular- es su aplicabilidad o utilidad para la vida, tanto si sus producciones son útiles en mayor o menor grado, como si resultan «al menos no perjudiciales» ${ }^{9}$. En todo caso Nietzsche ubica el argumento que cualquier "pensador» esgrimiría para sostener la validez de sus conceptos o creencias: «el conocimiento llegó a ser entonces parte de la vida, y en tanto vida, un poder creciente de continuo» ${ }^{10}$; pero lo describe precisamente para cuestionar las pretensiones de tales procedimientos y creencias, pues hace falta saber si la importante lucha instintiva que subyace al impulso de conocimiento y verdad deja o no elaborar un concepto de verdad intelectual que posea un auténtico «poder conservador de la vida»: «¿hasta qué punto soporta la verdad [tal] incorporación?» ${ }^{11}$.

La respuesta de Nietzsche se encuentra en su propia crítica: «quien era muy cuidadoso en la subsunción, tenía reducida probabilidad de seguir viviendo ${ }^{12}$. En este caso se refiere irónicamente a la fantasía de la lógica de alcanzar el mismo nivel de inmediatez que los instintos, no obstante se trata del mismo argumento que él usa para invalidar la lógica misma, a saber, que no se ajusta a la asociación inmediata de conservación. El razonar como tal estaría, por tanto, bastante alejado de su criterio principal, si este último se refiere a la «verdad» de la vida. En ello se cumple lo que expresa la descripción nietzscheana de la conciencia: «último y más tardío desarrollo de lo orgánico, [...], lo más inacabado y débil» ${ }^{13}$. La función vital la tienen los instintos, en tanto la especie queda garantizada sólo por su carácter asociativo inmediato: «si la asociación de conservación de los instintos no fuera sumamente más poderosa ni sirviera como reguladora en todo - en sus juicios [los de la conciencia] equivocados y su fantasear con los ojos abiertos, en

8 Cf. Nietzsche, $F W \S 110$, p. 469-470.

${ }^{9}$ Cf. Nietzsche, $F W \S 110$, p. 470.

10 «Die Erkenntnis wurde also zu einem Stück Leben selber und als Leben zu einer immerfort wachsenden Macht» (NIETzsche, FW §110, p. 471).

11 Nietzsche, FW \$110, p. 471.

12 Nietzsche, $F W$ §110, p. 471.

13 Nietzsche, FW $\S 11$, p. 382. 
su superficialidad y credulidad, incluso en su ser consciente-, la humanidad se habría ido a pique» ${ }^{14}$.

Ahora bien, justamente en tal carácter asociativo de los instintos puede residir una procedencia de la lógica distinta a la descrita por Nietzsche. De modo general, la ubica en lo ilógico, cuyo reino parece ser más vasto, de modo particular la localiza en «la propensión predominante a tratar lo semejante como lo igual - una propensión ilógica, pues en sí no hay nada que sea igual $-»^{15}$. Este sería por tanto el fundamento de la lógica, fundamento de suyo completamente ilógico en tanto «el decurso de los pensamientos y silogismos en nuestro cerebro actual corresponde a un proceso y a una lucha de impulsos, que por sí solos todos son ilógicos e injustos» ${ }^{16}$. En este planteamiento parece haber lugar, como hemos dicho antes, para una concepción en la que la «intuición de la vida» pueda ser descrita no sólo como «raíz metafísica de la lógica» — sea o no su resultado ilusorio-, sino también como lo más parecido a la realización de las tendencias de lo vivo, a saber, el impulso de ser «más vida» y «más-que-vida». Una caracterización de corte «pleonéxico» acuñada por Simmel en este contexto, que comporta, según él mismo, la impronta de la voluntad de poder del superhombre, conformando una de las dos dimensiones de la intuición vital: «El superhombre nietzscheano no es otra cosa que el nivel de desarrollo situado por encima del alcanzado por la humanidad en el momento presente, no es un meta fija que diera un sentido al desarrollo, sino la expresión de que no necesita ninguna otra que la vida en sí misma, esto es, posee su propio valor en el ser superado de cada nivel por uno más completo y desplegado» ${ }^{17}$. Tal movimiento proporcionaría, para Simmel, una fundamentación de una

14 NieTzsche, $F W \S 11$, p. 382.

15 NietZsche, $F W \S 111$, p. 471.

16 Nietzsche, $F W \S 111$, p. 472.

17 G. Simmel, Schopenhauer und Nietzsche: (SchNiet). Ein Vortragszyklus, en: GSG 10, 1995, p. 181. El «superhombre», por tanto, como fórmula del desarrollo de la humanidad sobre cada estadio dado. Sobre el sentido de actualización de la pleonexia sofista en la filosofía de la naturaleza del s. XIX, cf. H. Pfotenhauer, Die Kunst als Physiologie. Nietzsches ästhetische Theorie und literarische Produktion, Stuttgart: J.B. Metzlersche 1985, 73-75 pp. Al respecto informa también Solies, p. 178. 
lógica dinámica, en la que las formas no tienen carácter cualitativo fijo ni sustancial-fudacional.

\section{Vida y lógica dinámica (Simmel)}

Schopenhauer y Nietzsche se encuentran, para Simmel, justamente en el naciente significado teórico-ideológico (weltanschauliche) del concepto orgánico-metafísico-intuitivo de vida ${ }^{18}$. Continuidad (voluntad de vida) e individualidad (voluntad de poder) son los elementos schopenhauerianos y nietzscheanos, respectivamente, que hacen posible la definición lógico-dinámica que busca Simmel de la intuición vital. Schopenhauer sería «el primer filósofo moderno en preguntar no por el contenido de la vida, sino ¿qué es la vida, cuál es significado puramente como vida?». Voluntad, respuesta de Schopenhauer que suscribe Simmel ${ }^{19}$. Nietzsche, por su parte, habría encontrado, "partiendo de la vida como la única determinación de sí misma, como la única sustancia de todos sus contenidos, el externamente rehusado fin significante de la vida en la vida misma ${ }^{20}$, la cual, según su esencia, es desde sí misma elevación (Steigerung), devenir-más (Mehrwerden), desarrollo hacia la compleción y el poder, hacia la fuerza y la belleza, «de modo que en su propio progreso deviene más vida, ganando un valor que se eleva hacia el infinito» ${ }^{21}$. El punto nodal entre ambos filósofos sería por tanto el concepto de vida, cada uno situado en una de sus determinaciones fundamentales ${ }^{22}$, con lo que representarían posiciones unilaterales respecto a una completa intuición de la vida:

No estamos escindidos en vida sin límite y forma trazada, no vivimos parte en la continuidad y parte en la individualidad que se superan mutuamente. Más bien, la esencia fundante de la vida es precisamente aquella función unificada de suyo que denominé, de modo simbó-

\footnotetext{
18 Simmel, KmK (GSG 16), p. 188, o también Simmel, SchNiet (GSG 10), 187-189 pp.

19 Simmel, KmK (GSG 16), p. 188.

20 Simmel, KmK (GSG 16), p. 189.

21 SimMel, KmK (GSG 16), p. 189.

22 G. SimmeL, Lebensanschauung:(Leb) Vier metaphysische Kapitel, en: GSG 16, 1999, p. 229.
} 
lico y bastante incompleto, el trascender de sí misma, y que actualiza esto inmediatamente como una vida, lo que luego es desplegado, a través de sensaciones, suertes [y] conceptualidad en el dualismo de corrientes vitales [Lenbensströmungen] continuas y formas cerradas individualmente ${ }^{23}$.

Continuidad e individualidad permiten a Simmel ensayar una ampliación del concepto de vida que incorpore los elementos que conforman su posible (presunto) dualismo de origen. Describe por tanto la vida, en su «sentido absoluto», como:

[...] algo que se incluye a sí mismo en sentido relativo y a su contrario, del que es relativo y el cual es igualmente relativo a ella, o que se despliega diferenciada hacia ellos como sus fenómenos empíricos. Y por ello aparece la trascendencia de ella misma como el acto unitario del construir y quebrantar sus limitaciones, su alteridad, como el carácter de su absolutez - el cual hace perfectamente comprensible la digresión en contrarios autonomizados ${ }^{24}$.

En este sentido, lo que Simmel encuentra común en Schopenhauer y Nietzsche es la relación vida-voluntad de ambos planteamientos, "porque ellos conciben unilateralmente la autotrascendencia de la vida como conforme a voluntad (willensmäßig). Ella vale de hecho para todas las dimensiones del movimiento de la vida. Con ello la vida tiene dos definiciones complementarias entre sí: es más-vida y más-que-vida» ${ }^{25}$. La noción más original de Simmel debe su fuente, por tanto, a estas dos posiciones que encuentra complementarias. Más vida, porque en cada instante, así Simmel, arrastra algo hacia sí para transformarlo en vida; y más que vida, porque se produce un rebasarse (hinausschreiten) de ella sobre sí misma, una trasgresión de sus propios límites ${ }^{26}$.

Esta concepción conduce a Simmel hacia una «metafísica de la vida» que empieza a conformarse en el contexto de la discusión sobre la funcionalidad del pensar lógico-conceptual o discursivo

\footnotetext{
23 Simmel, Leb (GSG 16), p. 228.

24 Simmel, Leb (GSG 16), p. 228.

25 Simmel, Leb (GSG 16), p. 229.

26 Cf. Simmel, Leb (GSG 16), p. 229.
} 
(racionalismo moderno e intelectualismo) y de los elementos, criticados ya por Bergson, de una concepción mecánica, y conforme al entendimiento, de la vida ${ }^{27}$. La posición y crítica de Simmel se centran inicialmente en la rigidez y estancamiento del formalismo lógico-racional al intentar establecer una lógica con pretensión de validez absoluta. En este sentido los ensayos de Simmel procuran dar continuidad a la contraposición, implícita ya en Schopenhauer, Nietzsche, Bergson y Dilthey, entre lógica racional y lógica vital. De donde la diferencia más importante, sobre la que emprende su asimilación de la logicidad de la vida, consiste en la carencia de una continuidad real que unifique los elementos de la lógica formal en torno a «direcciones» $\mathrm{O}$ «rítmicas», aunque sin fin concreto ${ }^{28}$. Ahora bien, tal continuidad es precisamente el modo de ser de la vida en tanto imposible de ser conceptualizada o concebida, esto es, en tanto su dinamismo implica operaciones lógico-dialécticas en las que ella logra realizarse como tal sólo si penetra y se convierte en

27 Específicamente destaca Simmel la observación de Bergson «de que nuestra lógica casi es directamente lógica de cuerpos fijos. Esencialmente ella se funda - exactamente como el mecanismo - en los conceptos básicos de identidad y ser-otro [...] No obstante la contraposición de identidad y ser-otro desaparece en la viva 'continuidad del transformarse'», en: G. Simmel, Zur Philosophie der Kunst. Philosophische und kunstphilosophische Aufsätze:(Phk), Gertrud Simmel (ed.), Postdam: G. Kiepenheuer 1922, 126-145 pp.

28 G. Simmel, Fragmente und Aufsätze:(FrA) Aus dem Nachlaß und Veröffentlichungen der letzten drei Jahre, G. Kantorowicz (ed.), München 1923 (reimpreso Hildesheim 1967), 97-105 pp. Para un informe detallado de los pasajes de la obra de Simmel en los que su crítica a la lógica racional adquiere mayor contundencia, cf. P. Christian, Einheit und Zwiespalt. Zum hegelianisierenden Denken in der Philosophie und Soziologie Georg Simmels, Diss. Duncker und Humblot (=Sozilogische Schriften, 27), Berlin 1978, 62-63 pp. Añado la expresión «sin fin concreto» en el sentido kantiano de «conformidad a fin sin fin»-expuesto en el contexto de la analítica de lo bello (tercer momento: juicios de gusto) - , dado que las «formas lógicas» $\mathrm{y}$ «direccionalidades» del planteamiento de Simmel parecen conservar esa misma configuración, a saber, la de una acción teleológica sin concepto previo o sin representación de un fin: «un objeto o un estado del ánimo, o también una acción, dícense conforme a fin, aún cuando su posibilidad no presuponga necesariamente la representación de un fin», en: Immanuel Kant, Crítica de la facultad de juzgar:(CFJ), trad. P. Oyarzún, Caracas: Monte Ávila 1991, §10, p. 126. Haciendo la salvedad no obstante, de que Simmel no ubica - como Kant- esta posibilidad en el «libre juego de las facultades», sino más bien en el impulso vital. En otro lugar he comentado las implicaciones epistemológicas de la «conformidad a fin sin fin» kantiana, cf. R. Franco: «La imaginación como criterio del entendimiento», en: M. C. Paredes (ed.): Kant... Salamanca, p. 141ss. 
su negatividad. En expresión de Simmel la vida se realiza en tanto «se convierte en no-vida» ${ }^{29}$.

Este enfoque inicial plantea al menos dos problemas fundamentales. Uno es el de la posibilidad de establecer un concepto de vida, dado que su intuición implica concebirla como tal, sin que sea efectivo ni posible determinarla a través - o como - una lógica formal-conceptual. El siguiente problema, suponiendo que lo anterior sea posible, se referiría a las dificultades de comprender una dialéctica que tome como referencia sólo una de las partes de su síntesis, en tanto el concepto de vida que de ello resulte quedará siempre en segundo plano, pues la referencia final, e incluso definitoria de la vida constituiría una realidad en la que la vida sólo cumpliría la función de ser referente explicativo de lo que realmente opera en la realidad. Al parecer, tales dificultades sólo pueden ser asimiladas en tanto significados o plataformas de una «especie de ontología de la vida» cuya verdadera referencia no sea exactamente la vida como tal, sino más bien el dinamismo peculiar de la subjetividad ${ }^{30}$.

En este sentido, pese a que Simmel critica la racionalidad de la lógica, reconoce que sus propias reflexiones sobre la vida plantean dificultades lógicas que sólo pueden ser comprendidas como procedentes de un estrato del que se nutre la lógica misma en tanto lógica de lo instintivo y consciente a la vez, que ejecuta operaciones aún más complejas ${ }^{31}$. Teniendo en cuenta este presupuesto, procuraremos reconstruir el procedimiento de Simmel en el primer capítulo (Transzendenz des Lebens) de su ensayo, guiándonos por la secuencia de intuiciones especulativas que dan lugar a la fundamentación metafísica de una lógica general, en tanto determinada por la estructura y operatividad de la vida como tal.

29 Simmel, PhK, (cf. n. 27), p. 138.

30 En lo que sigue atenderemos más al procedimiento de Simmel, que lo conduce hacia tal planteamiento, que a un ensayo exhaustivo de resolución de estas dificultades.

${ }^{31}$ Respecto a la convicción de Simmel de que su modo de pensar la vida implica la «raíz metafísica de la lógica» se echa de menos una explicación más detallada de la misma, ya que sólo se encuentra enunciada al final del primer capítulo de Lebensanschauung, dando a entender con ello que tal acepción queda de alguna manera ya expuesta en el mismo. 


\section{Tiempo - Límite - Autoconciencia}

El ensayo de Simmel parte del carácter de contradicción del tiempo, el cual, como hemos anticipado, encuentra su realidad sólo en una especie de ontología de la vida ${ }^{32}$. Pero ésta, paradójicamente, no es una realidad existente de suyo, ya que su verdadera existencia o «forma fáctica más simple y fundamental» (einfachste und grundlegende Tatsacheform) es la autoconciencia, la cual constituye - así Simmel - «el fenómeno originario del espíritu en cuanto un humano-viviente en general» ${ }^{33}$. La pretensión de Simmel es, por tanto, describir la intuición de lo que él denomina «categoría» del «rebasarse de la vida a sí misma» ${ }^{34}$, la cual, en un primer momento, mientras no se analice la «forma fáctica fundamental» (la autoconciencia), sirve como presuposición o «símbolo» inicial: «solamente comprendida en su esencia, la considero [a la categoría] en todo caso como enteramente primaria» ${ }^{35}$.

Simmel incorpora la reflexión sobre el tiempo y la autoconciencia como punto de partida y exposición, respectivamente, de su concepción de vida como contradicción del constante permanecer en sí y transcenderse a sí misma, lo cual, en un primer momento, encuentra su mejor expresión en el comportamiento del conocer consigo mismo, a saber, sentar y superar su limitación inmanente:

[...] que nosotros como esencia cognoscente y dentro de las posibilidades del conocer mismo podemos captar la idea general de que el mundo no cabría dentro de las formas de nuestro conocer; que nosotros, sólo de manera completamente problemática, podemos pensar una condición del mundo, que igualmente no podemos pensar, [todo ello] es un traspasar de la vida espiritual sobre sí misma, avance y allendidad no solamente de un singular, sino de su límite en general,

32 Sobre este aspecto puede decirse que Simmel se aleja de su conocida influencia kantiana, en el sentido de la pretensión de una subjetividad atemporal. Para una observación al respecto, cf. J. Ferreira, «Da vida ao tempo: Simmel e a construção da subjetividade no mundo moderno», Revista Brasileira de Ciências Sociais 15-44 (2000), p. 104ss.

33 Simmel, Leb (GSG 16), p. 223.

34 Simmel, Leb (GSG 16), p. 223.

35 SimmeL, Leb (GSG 16), p. 223. 
un acto de la autotrascendencia que sienta primeramente - tanto si es real-efectiva como sólo posible- el límite mismo $^{36}$.

El tiempo, en tanto presente carente de temporalidad, permite a Simmel visualizar la funcionalidad de esta contradicción constitutiva del concepto de límite (un ya-no y todavía-no): «presente [...] significa exclusivamente el colisionar entre pasado y futuro» ${ }^{37}$. Tal característica permite a Simmel situar la reflexión sobre el tiempo en un ámbito lógico-metafísico-espiritual. Lógico en tanto descripción teleológico-dialéctica de movimientos de un proceso; metafísico-espiritual en tanto tal proceso pertenece a una totalidad que se autotrasciende y que posee carácter de autoconciencia. Para estos efectos el presente, en tanto presente vital, posee la misma estructura que «la voluntad en acto»: «El 'fin', de algún modo remoto, aparece como un punto fijo separado discontinuamente del presente, mientras que lo decisivo, concretamente el vivir inmediato de la voluntad $-\mathrm{y}$ del sentir, y del pensar-, está en el futuro: el presente de la vida consiste en lo siguiente: en que trasciende el presente ${ }^{38}$.

De la estructura y movimiento de la voluntad infiere Simmel que el presente como tal no es real y que, por tanto, la única extensión posible del tiempo la representa el movimiento de la vida: «la vida es efectivamente pasado y futuro [...], el modo de existencia peculiar, para cuya facticidad no es válida esta separación [...], sólo para la vida es el tiempo real» ${ }^{39}$. Esta importante sentencia plantea una serie de implicaciones en las que resulta difícil no extraer de modo casi definitivo un concepto de tiempo real, concebido sólo a partir de la intuición de la vida.

36 SimmeL, Leb (GSG 16), 216-217 pp. Al respecto cabe mencionar la observación de J. Ferreira en la que estos aspectos quedan recogidos en el ejemplo del proceso de cognición: «A cognição pode fornecer um exemplo de como a vida se estrutura de modo transcendental, isto é, tanto a partir do reconhecimento de um limite, de uma fronteira, quanto de um tipo particular de transgressão a essa restrição», en: Ferreira (cf. n. 32), p. 110.

37 Simmel, Leb (GSG 16), p. 218. Para un análisis completo del ensayo de Simmel en el contexto de sus implicaciones onto-temporales, históricas y sociológicas cf. FerreIRA, 103117 pp.

38 SimMEL, Leb (GSG 16), p. 220.

39 Simmel, Leb (GSG 16), p. 221 (cursiva mía). 
Ahora bien, aunque este parezca el momento oportuno para culminar la secuencia intuitiva que se pretende mostrar en Lebensanschuung, Simmel sugiere darle continuidad hasta un modo de existencia en el que se cumpla una verdadera, en tanto posible y real, trascendencia, es decir en tanto los momentos de la vida no se agoten en la simple reproducción de vida - lo que, en último término, permanece dentro del ámbito de la identidad - , sino que ella misma se trascienda en tanto intente dar un salto, si se quiere, ontológico. Esta realidad se da de hecho, según Simmel, en la autoconciencia, con ella entra esta idea de la vida en el ámbito fenoménico, existente de modo real-efectivo ${ }^{40}$. En este sentido, la estructura de la autoconciencia (saberse-a-sí-mismo infinito) expresa y ejecuta el movimiento vital con todas sus implicaciones.

En tanto el yo no solamente se contrapone a sí mismo o se constituye a sí mismo, en tanto sapiente, objeto de su propio saber, sino que también se juzga como un tercero, se estima o desestima, y con ello se sitúa también sobre sí mismo, se sobrepasa a sí mismo permanentemente y permanece no obstante en sí mismo, porque su sujeto y objeto son idénticos; esta identidad se separa, sin desgarrarse - ya que no se trata de ninguna sustancialista fija - en el proceso de la vida espiritual del saberse a sí mismo ${ }^{41}$.

Se trata por tanto, de una autotrascendencia continua que se ejecuta de modo real en la autoconciencia como el «fenómeno originario del espíritu». Ahora bien, esta estructura - pese al modo de exposición «dialéctico» de lo que Simmel llama significados «categoriales» de la vida ${ }^{42}$ - no recoge un tránsito entre grados de

40 Cf. Christian (cf. n. 28), p. 90.

41 Simmel, Leb (GSG 16), p. 223.

42 P. Christian comprende el concepto de vida de Simmel como continuación de la determinación de la vida expuesta por Hegel en los Frühschriften, a saber, «Die Verbindung der Verbindung und Nichtverbindung», la cual subyace en dos planteamiento claves de Simmel: «Selbstbewußtsein als einem Symbol oder realen Selbstausdruck des Lebens und zweitens seiner Konzeption von der Todesverflochtenheit des Lebens» (cf. Christian, p. 87). Una discusión sobre el pensamiento 'hegelianizante' de Simmel, propugnado por Christian, se encuentra en: P.E. SchNABEL, «Positivismus, Ästhetizismus, Impressionismus, Hegelianismus. Simmel-Renaissance in der Sackgasse?», en: H. J. Dahme y O. Rammstedt (eds), Georg 
trascendencia o significados de la vida, sino más bien expresa una especie de ontología fundada en una doble operatividad, posible en distintos planos de la intuición, sin que se produzca necesariamente un paso de uno a otro. Por un lado tenemos la vida en tanto proceso de lo orgánico-inorgánico, que «quiere» ser «más-quevida», y por otro, el de la autoconciencia como espíritu viviente cuyo movimiento intelectual ejecuta realmente la voluntad que la estructura de la vida deja intuir, bien sea en su carácter dualista unificado, temporalidad, carácter de límite puesto y superado, etc. Una ontología que, mediada inicialmente por el reconocimiento de nuestra peculiaridad temporal - saber este que a su vez depende de la «verdadera forma fáctica» (la autoconciencia) de ejecución de sus condiciones - , concluye con la dinamicidad de un proceso de autoconcepción y realización de una entidad viva. Implicaciones que bien podrían ser tenidas como antecedentes de lo que luego Heidegger comprenderá bajo el registro de existenciario ${ }^{43}$. Tema que sugiere una investigación aparte ${ }^{44}$.

Simmel und die Moderne..., Frankfurt a. M 1984, 282-317 pp.

43 Gadamer hace hincapié en la caracterización simmeliana de la vida, en cuanto relación orgánica de la vivencia con la totalidad de la vida, como «un estar volcada de la vida hacia algo que va más allá de sí misma» (cita a Lebensanschauung), lo cual representa un punto de partida evidente para la circunscripción ontológico-dialéctica de la vida en Heidegger, cf. H. G. Gadamer, Verdad y Método I, Salamanca: Sígueme 1996, p. 106, n. 57. Informa sobre la admiración de Heidegger por los últimos escritos de Simmel como un reconocimiento personal a la personalidad filosófica de Simmel y como impulso a cuestiones de contenido en el mismo Heidegger. En lo referente a nuestra lectura, inicialmente nos mostramos en desacuerdo con la afirmación de Gadamer de que «sólo Heidegger [de entre Nietzsche, Bergson y Simmel] ha llegado a hacer consciente de una manera general la radical exigencia que se plantea al pensamiento con la inadecuación del concepto de sustancia para el ser y el conocimiento histórico» (p. 306). Para ello tenemos en cuenta la concepción simmeliana de la estructura de la autoconciencia como ejecución del movimiento vital con todas sus implicaciones. Retomamos un fragmento antes citado (cf. n. 41): «esta identidad se separa, sin desgarrarse - ya que no se trata de ninguna sustancialista fija -, en el proceso de la vida espiritual del saberse a sí mismo» (Simmel, Leb [GSG 16], p. 223).

44 También el enfoque inferencialista de R. Brandom podría ser tenido como 'heredero' actual de estos planteamientos. Su proyecto parte de una semántica pragmática en la que se analiza el modo inferencial en que son articulados los informes no inferenciales, motivado por el mecanismo de dar y pedir razones, lo que permite, p.e., exponer relaciones entre normatividad y razonamiento práctico, así como las bases de la expresividad que hacen posible la objetividad del contenido conceptual (cf. R. Brandom, La articulación de las razo- 
Provisionalmente cabe aproximar la conclusión de que el concepto nietzscheano de intuición, y sus implicaciones en la determinación de la validez de lo conceptual, ofrece a Simmel recursos para una ampliación de sus resultados epistemológicos. Los conceptos, las formas, la lógica en general, obtendrían una impronta vital que socavaría su tendencia a la rigidez designadora y fundacional, contraria al carácter dinámico de lo que refieren. O. Rammstedt y M. Cantó logran expresarlo de un modo que sin duda agradaría a Simmel: «[...] las formas son cristalizaciones del incontenible fluir de la vida [...] la vida se convierte en arte o ciencia, por ejemplo [...]. Siguiendo esta perspectiva tendríamos que entender el arte como un modo de expresar la vida a través de formas (artísticas, estéticas) ${ }^{45}$.

\section{RESUMEN}

Las nociones nietzscheanas de intuición y voluntad de poder $-\mathrm{y}$ sus implicaciones en la determinación de la validez de lo conceptual- ofrecen a Simmel recursos para una ampliación de sus resultados epistemológicos. El carácter autotrascendente del concepto de vida, en correspondencia con la autosuperación del superhombre, imposibilita una definición estable de la vida, lo cual repercute en los registros en los que ésta logra plasmarse. Los conceptos, las formas, la lógica en general, obtendrían una impronta vital que socavaría su tendencia a la rigidez designadora y fundacional.

nes, Madrid: Siglo XXI 1002, 46-47pp.). Una visión analítica situada en los mecanismos de autoconstitución de la autoconciencia que tienen que ver con la constante reformulación de la propia concepción de sí (cf. R. BRAnDom, «Selbstbewusstsein und Selbst-Konstitution. Die Struktur von Wünschen und Anerkennung», en: C. Halbig, M. Quante, L. Siep (eds), Hegels Erbe, Frankfurt a.M: Suhrkamp 2004, p. 46ss).

45 O. Rammstedt y M. Cantó, «Georg Simmel (1858-1918)», en O. Sabido Ramos, Georg Simmel. Una revisión contemporánea, Barcelona/México 2007, p. 123, cursiva mía. Jankélévitch, en su discusión con Rickert, lo plantea de modo autotrascendente, en tanto constante superación de límites, con la siguiente interrogante: «La Selbsttranszendenz de Simmel, la Selbstüberwindung de Nietzsche, es ya una forma [...] una determinación positiva como cualquier otra, y el hecho de sobrepasar y destruir cualquier forma - ¿no es, para la vida, tener una forma?», en: JANKÉLÉVITCH (cf. n. 6), p. 87. 
Palabras clave: Intuición; forma; voluntad; autotrascendencia; vida.

\section{ABSTRACT}

The Nietzschean notions of intuition and will to power and its implications in determining the validity of the conceptual offer Simmel the resources for an expansion of its outcome of his epistemological results. The self-transcendent character of the concept of life, in correspondence with the self-overcoming of the great man, make impossible a stable definition of life, which has consequences for the records in which it succeeds in shape. The concepts, ways, the logic in general, would get a vital stamp that would undermine its trend of designating stiffness and foundational.

Key words: Intuition; form; will; self-transcendence; life. 
